Chugai, Eisai, Taisho Toyama, UCB, Janssen, Eli Lilly, and NIPPON KAYAKU., Speakers bureau: Astellas, BMS, Daiichi-Sankyo, Mitsubishi Tanabe, Pfizer, Ayumi, Takeda, Chugai, Eisai, Taisho Toyama, UCB, Janssen, Eli Lilly, and NIPPON KAYAKU. DOI: 10.1136/annrheumdis-2019-eular.4714

\section{THU0221 DRY EYE IN SJÖGREN'S SYNDROME: CHEMOKINE AND CYTOKINE TEAR SPECTRUM}

Gabriela Hernandez-Molina, Narlly Ruiz-Quintero, Guadalupe Lima, Diego Hernández-Ramírez, Amaya Llorente-Chavez, Vanessa SaavedraGonzález, Rodolfo Jimenez-Soto, Luis Llorente. Instituto Nacional de Ciencias Médicas y Nutrición Salvador Zubirán, Mexico City, Mexico

Background: Previous studies have linked the participation of multiple chemokines and cytokines in the physiopathology of primary Sjögren's syndrome (PSS), however data regarding their presence in tears is scarce

Objectives: To evaluate a panel of chemokines/cytokines in the tears of patients with PSS and correlate them with ocular symptoms as well as objective ocular tests.

Methods: We included 21 patients with PSS (EULAR/ACR criteria). A single expert ophthalmologist in dry eye evaluated the patients and assessed the tear film break-up time, Schirmer-I test, tear meniscus height, the Van Bijsterveld staining score and the SICCA Ocular Staining Score (OSS). We classified lacrimal dysfunction severity in two categories ( $1=$ mild, mild $/$ moderate or moderate, and $2=$ moderate/severe and severe). We scored the ESSPRI, and ocular dryness VAS as well as the Ocular Surface Disease Index (OSDI), a 12-item scale for the assessment of symptoms related to dry eye disease and their effect on vision. Tear samples were collected using sterile tear flow strips, that were immediately frozen at $-86^{\circ} \mathrm{C}$ until assayed. Once defrosted, the tears were extracted from the strips using a buffer containing $0.5 \mathrm{M} \mathrm{NaCl}$ and $0.5 \%$ Tween-20. We tested IFN- $\gamma$, IL-10, IL-12, IL-17A, IL-1 $\beta$, IL-2, IL-21, IL-23, IL-5, IL-6, IL-8, TNF- $\alpha$, BAFF, CXCL10 and CCL2 by Luminometry. We also included 21 healthy controls without dry eye, to test chemokines/ cytokines that after our initial screening were meaningful.

Results: Most patients were females (90.4\%), mean age $59.3 \pm 13$ years and median disease duration 7.9 years $(0.5-27)$. All of them had ocular and oral symptoms. The median tear film break-up time was 6 seconds (2-9), median Schirmer-I test $6 \mathrm{~mm}$ (1-25), median lacrimal meniscus height $1.5 \mathrm{~mm}(0.5-2)$, median Van Bijsterveld staining score 10 points (2-18), median OSS 7 points (2-11), median ESPPRI score 6.7 points (29.2) and median ocular dryness EVA score 9 points (1-10).

We did not detect most of the evaluated chemokines/cytokines with the exception of IL-8, CXCL10, and CCL2. The former was similar in both, patients and controls. PSS patients had lower levels of CXCL10 (472.8 $\mathrm{pg} / \mu \mathrm{L}$ vs $1652 \mathrm{pg} / \mu \mathrm{L}, \mathrm{p}=0.01)$ and $\mathrm{CCL} 2$ (1.08 $\mathrm{pg} / \mu \mathrm{L}$ vs $9 \mathrm{pg} / \mu \mathrm{L}$ ) than controls. Indeed, patients with worst lacrimal dysfunction severity had the lowest levels of CXCL10 (239.3 pg/ $\mu \mathrm{L}$ vs. $646.2 \mathrm{pg} / \mu \mathrm{L}, \mathrm{p}=0.02)$. We found correlations among $\mathrm{CXCL} 10$ and $\mathrm{CCL} 2(\mathrm{~T}=0.30, \mathrm{p}=0.02)$ and lacrimal meniscus height $(T=0.55, p=0.005)$, as well as with CCL2 and lacrimal meniscus height $(T=0-57, p=0.01)$. None of the other variables were correlated.

Conclusion: We identified CXCL10 and CCL2 as the main chemokines in tears of patients with PSS. CXCL10 seems to participate in the normal eye homeostasis.

Disclosure of Interests: None declared

DOI: 10.1136/annrheumdis-2019-eular.5226

\section{THU0222 PLASMACYTOID DCS FROM PATIENTS WITH SJÖGREN'S SYNDROME ARE TRANSCRIPTIONALLY PRIMED FOR ENHANCED PRO-INFLAMMATORY CYTOKINE PRODUCTION}

Maarten Hillen ${ }^{1}$, Aridaman Pandit ${ }^{1}$, Sofie Blokland ${ }^{1}$, Sarita Ay Hartgring ${ }^{1}$, Cornelis Bekker ${ }^{1}$, Eefje van der Heijden ${ }^{1}$, Nila Servaas ${ }^{1}$, Marzia Rossato ${ }^{1}$, Aike A. Kruize ${ }^{2}$, Joel van Roon ${ }^{1}$, Timothy R. Radstake ${ }^{2} .{ }^{1}$ University Medical Centre Utrecht, Laboratory of Translational Immunology/Rheumatology and Clinical Immunology, Utrecht, Netherlands; ${ }^{2}$ University Medical Centre Utrecht, Rheumatology and Clinical Immunology, Utrecht, Netherlands

Background: Type-I IFN activity is associated with pathogenesis and increased disease activity in primary Sjögren's syndrome (pSS). In addition, deficiency for the type-I IFN receptor in mice prevents experimentalSjögren's syndrome. Plasmacytoid dendritic cells (pDC) are the premier type-I IFN producing immune cells and aberrances in their functional properties may underlie pSS immunopathology. Assessing the molecular basis of this may provide a better understanding of pSS pathogenesis and new opportunities for therapeutic intervention.

Objectives: To delineate the dysregulation of pSS pDCs using RNA sequencing and compare their transcriptional profile to pDCs obtained from patients with non-Sjögren's sicca (nSS) and healthy controls (HC).

Methods: All pSS patients met the classification criteria. nSS patients presented with dryness complaints without a known cause, did not have any generalized autoimmune disease including pSS as evaluated by an experienced rheumatologist, and did not fulfil the classification criteria. pSS $(n=25)$, nSS $(n=20)$, and HC $(n=17)$ donors were included in two independent cohorts $(n=31$ each). Circulating BDCA-4 expressing pDCs were isolated and RNA-sequencing was performed, after which data-driven networks and modular analysis were used to identify signatures of consistently differentially-expressed genes. pSS and $\mathrm{HC}$ pDCs were cultured in the presence of endosomal TLR ligands, after which gene expression and secreted cytokine levels were measured.

Results: We identified signatures of consistently co-expressed and differentially expressed genes that indicated transcriptional activation in patien pDCs, which was remarkably reproducible in two independent cohorts These included a type-I IFN-associated signature, a ribosomal protein sig nature, and a transcriptional machinery signature. Corroborating the transcriptomic profile, stimulated pSS pDCs produced higher levels of typeinterferon upon in vitro stimulation. nSS patients formed an intermediate group in which some patients were molecularly similar to pSS patients. Finally, we developed a discriminative classifier on the basis of the identified transcriptional profiles that discriminated pSS patients from $\mathrm{HC}$ with $100 \%$ sensitivity and $80 \%$ specificity, and identified a group of pSS-like patients within the nSS group.

Conclusion: Circulating pSS pDCs exhibit a transcriptional signature similar to activated pDCs and are primed for enhanced production of proinflammatory cytokines, including type-I IFN. Our data provide in-depth characterization of the aberrant regulation of pSS pDCs and substantiate their perceived role in the immunopathology of pSS and other type-I interferon-associated autoimmune diseases.

Disclosure of Interests: None declared

DOI: 10.1136/annrheumdis-2019-eular.7019

\section{THU0223 CHRONIC ADRENERGIC STIMULATION OF MINOR SALIVARY GLANDS OF PATIENTSWITH PRIMARY SJÖGREN'S DRIVES ER STRESS AND ACTIVATION OF THE UNFOLDED PROTEIN RESPONSE}

Kalliopi Moustaka ${ }^{1}$, Stergios Katsiougiannis ${ }^{1}$, Roxane Tenta ${ }^{1}$, Sofia Havaki ${ }^{2}$ Pari Koutsoudaki ${ }^{2}$, Haralampos M. Moutsopoulos ${ }^{3}$, Fotini Skopouli ${ }^{1}$. Harokopio University, Nutrition and Clinical Dietetics, Ko $\alpha \lambda \lambda_{1} \theta \dot{\varepsilon} \alpha$, Greece; ${ }^{2}$ National and Kapodistrian University of Athens, Histology and Embryology, Athens, Greece; ${ }^{3}$ The Academy of Athens, Athens, Greece

Background: Sjögren's syndrome (SS) is a common autoimmune disease in which the main targets of immune injury are specific secretory epithelia, such as salivary and lacrimal glands. Stress appears to play a significant role in the initiation of this entity.

Objectives: The aim of the present study was to investigate whether chronic stress plays a role in triggering endoplasmic reticulum (ER) stress in salivary gland epithelial cells from SS patients.

Methods: Minor salivary gland biopsy specimens were obtained from six SS patients and six control patients with sicca symptoms not fulfilling AECG criteria [1]. The expression and cellular localization of $\beta 1-, \quad \beta 2$ and $\alpha 1$-adrenoceptors and the levels of cAMP were measured by immunofluorescence. The morphology of the ER was evaluated in situ by Transmission Electron Microscopy (TEM). Primary salivary gland epithelial cell lines (SGEC) derived from minor salivary gland biopsies, were established by the explant out-growth technique [2] and were treated with epinephrine and norepinephrine. The protein levels of the ER stress markers GRP78/Bip and C/EBP homologous protein (CHOP) were determined by immunoblot analysis.

Results: In situ immunofluorescence staining revealed increased expression of $\beta 1-, \beta 2$ - and $\alpha 1$ adrenoceptors as well as cAMP levels in tissues derived from SS patients compared to controls. TEM evaluation of salivary tissues from SS subjects revealed extensive dilation of the ER lumen compared to controls. Treatment of SGEC with epinephrine and norepinephrine did not influence cell survival (cell viability assay). To mimic chronic stress in vitro, epinephrine was applied for 10 days on SGEC. It was found that $20 \mu \mathrm{M}$ Epinephrine induced severe ER stress on SGEC, as attested by increased expression of GRP78/Bip and CHOP, after 3 and 6 days of treatment. The expression of ER stress markers returned to basal levels after 10 days of treatment. 